This is a post-peer-review, pre-copyedit version of an article published in Brain Topography. The final authenticated version is available online at: https://doi.org/10.1007/s10548-019-00737-5

\title{
The role of the temporoparietal junction in self-other distinction
}

\section{Authors}

François Quesque ${ }^{1,2}$ and Marcel Brass ${ }^{1}$

\section{Affiliations}

${ }^{1}$ Department of Experimental Psychology, Ghent University, Henri Dunantlaan 2, B-9000, Belgium

${ }^{2}$ CMRR, Inserm U1171, Lille

\section{Corresponding author}

Marcel Brass: marcel.brass@ugent.be 


\begin{abstract}
Being able to discriminate between what originates from ourselves and what originates from others is critical for efficient interactions with our social environment. However, it remains an open question whether self-other distinction is a domain-general mechanism that is involved in various social-cognitive functions or whether specific 'self-other distinction mechanisms' exist for each of these functions. On the neural level, there is evidence that self-other distinction is related to a specific brain region at the border of the superior temporal and inferior parietal cortex, the temporoparietal junction (TPJ). Demonstrating that the TPJ plays a role in social processes that require self-other distinction would support the idea of a domain-general mechanism of self-other distinction. In the present paper, we review evidence coming from clinical observations, neuroimaging experiments and a meta-analysis indicating the involvement of the TPJ in various cognitive operations requiring self-other distinction. At the perceptual level, we discuss the human ability to identify one's own body and to distinguish it from others. At the action level, we review research on the human ability to experience agency and the control of imitative response tendencies. Finally, at the mental-state level, we discuss the ability to attribute mental states to others. Based on this integrative review, we suggest that the TPJ, and in particular its dorsal part, supports a domain general ability to enhance task-relevant representations when self-related and other-related representations are in conflict. Finally, this conception allows us to propose a unifying architecture for the emergence of numerous sociocognitive abilities.
\end{abstract}


Key words: Temporoparietal Junction, TPJ, Self-Other Distinction, Theory of Mind, Agency 


\section{Introduction}

Being able to distinguish what originates from ourselves and what originates from others is critical when interacting with our social and non-social environment (Jeannerod, 2003). First, at the most fundamental, the perceptual level, it allows us to have an accurate representation of our body, its present characteristics and its boundaries (sense of ownership). Second, at the motor level, being able to distinguish sensory consequences of our own actions from sensory events produced by other people's behavior constitutes the minimal setting to experience the sense of agency over our own actions and adapt our behavior to its direct environmental constraints (Jeannerod, 2003). Alterations of the sense of agency might be central to psychotic disorders and hallucinations (Blakemore, Smith, Steel, Johnstone \& Frith, 2000; Daprati et al., 1997). Finally, at the mental representation level, distinguishing between our own and other's beliefs, desires and intentions allows us to navigate efficiently in the social world. Self-other distinction seems therefore to be a necessary component for a wide range of social-perceptual, motor-cognitive and social functions. This raises the crucial question whether self-other distinction constitutes a domain-general mechanism common to all these different functions or whether multiple mechanisms for self-other distinction exist? A neural recycling approach (Corbetta, Patel \& Shulman, 2008) might favor a single mechanism that has developed on a basic sensorimotor level and then extends to more complex levels of mental state representation (Brass, Ruby \& Spengler, 2009). Alternatively, specific modules allowing to distinguish between self and other may exist for each kind of psychological function such as perception, agency and mental state 
representation. One way to address this question is to investigate whether self-other distinction in different domains recruits different or similar neural resources (Happé, Cook \& Bird, 2017).

A potential candidate brain region for a domain-general self-other distinction mechanism is the temporoparietal junction (TPJ) that has been linked to various cognitive operations requiring self-other distinction (Farrer, Franck, Georgieff, Frith, Decety \& Jeannerod, 2003; Saxe \& Kanwisher, 2003; Sperduti, Delaveau, Fossati \& Nadel, 2011; Uddin Molnar-Szakacs, Zaidel \& Iacoboni, 2006). It is however important to keep in mind that the TPJ has not only been related to social-cognitive functions requiring self-other distinction but rather to a large range of psychological functions (Bukowski \& Lamm, 2017). This brain region is for example commonly discussed as playing a central role in attention reorientation (e.g. Corbetta \& Shulman, 2002; Serences, Shomstein, Leber, Golay, Egeth \& Yantis, 2005), language processing (e.g. Just, Carpenter, Keller, Eddy \& Thulborn, 1996; Pulvermüller, 2005), episodic memory (e.g. Cabeza, Ciaramelli \& Moscovitch, 2012), as well as in a vast variety of social cognition tasks ranging from moral judgements (e.g. Young, Camprodon, Hauser, Pascual-Leone \& Saxe, 2010) to mental states' ascription (e.g. Lombardo et al. 2010; Saxe \& Kanwisher, 2003). Given the lack of specificity that currently exist in the neuroscientific literature regarding TPJ function, it is an open question whether TPJ may constitute the neural substrate of a domain-general self-other distinction mechanism. In the present paper, we aim to summarize the involvement of TPJ in different social-perceptual, motor cognitive and mental representational functions that require self-other distinction. To achieve this goal, we will first define the TPJ on a neuroanatomical level. We will then discuss different kinds of psychological processes relying on the distinction between self and other and confront their respective neurological substrate. Finally, on the basis 
of this review, we will propose an integrative view concerning the role of the temporoparietal junction in self-other distinction.

\section{Intrinsic and extrinsic heterogeneity within the concept of TPJ}

The temporoparietal junction (TPJ) is located at the border of the posterior superior temporal and inferior parietal lobes. Importantly, despite its common use in the neuroscience literature, its description remains largely unspecific, not mapping precisely onto any specific anatomical landmarks (Carter \& Huettel, 2013). The large range of psychological process supposedly linked to the TPJ could then be a mere consequence of its fussy anatomical definition. An anatomical definition that takes into account the lack of clear anatomical landmarks, was put forward by Bukowski and Lamm (2017). They proposed to imagine a straight line connecting the end of the lateral sulcus (Sylvian fissure) to the rostro-lateral edge of the occipital cortex (see Figure 1, dashed red line). This imaginary line would represent the strict delimitation between the temporal and parietal lobes and the brain area directly surrounding it constitutes the "temporoparietal junction" (red ellipse). The region located above the imaginary line will be referred to as the dorsal part of the TPJ, and the region located below as the ventral part. The dorsal part of the TPJ would then encompass the more ventral part of the supramarginal gyrus (SMG, represented in orange) and the ventral part of the angular gyrus (AG, represented in green). The ventral part of the TPJ consists of the most dorsal extent of the superior temporal gyrus (STG, represented in cyan) and the most posterior extents of the superior temporal sulcus (STS, represented in pink). Hence, the TPJ encompass many brain structures and, consequently, receives input from numerous others, including the lateral and posterior thalamus, the lateral 
anterior, medial anterior and ventral prefrontal cortex, the anterior insula, as well as the visual, auditory, somesthetic, and limbic areas (Bzdok et al. 2013; Mars, Sallet, Schüffelgen, Jbabdi, Toni \& Rushworth, 2012; Nieuwenhuys, Voogd \& Van Huijzen, 2007).

\section{// Insert Figure 1 around here //}

As it is the case for most cortical structures, the TPJ is bi-laterally present. However, some lateralization of specific functions has been reported in the literature (e.g. Saxe \& Kanwisher, 2000). Given the wide range of brain structures that is encompassed under this denomination, differentiation between anterior and posterior, as well as between ventral and dorsal parts of the TPJ should also be considered when interpreting brain imaging evidence. As pointed by Bzdok et al. (2013), the lack of specificity of the anatomical definition, in addition to the inclusion of findings based on relatively small sample sizes may have contributed to the heterogeneity of previous findings concerning the precise role of the TPJ. The aim of the present paper is not to discuss the wide range of functions that have been attributed to the TPJ. Rather we would like to focus on its potential role in self-other distinction.

\section{The many levels of self-others distinction}

A first necessary step to discuss the neural substrates of self-others distinction is to define to which specific theoretical construct the term "self" refers, as it has been used to discuss multiple different cognitive phenomena (Uddin, 2011). From a social psychological point of view, it has been proposed that people have access to five different levels of information about themselves, which would develop during different periods of ontogeny, and emerge from 
multiple domains ranging from perceptual to cultural experiences (Neisser, 1995). The "ecological self" is the lowest level and constitutes the integration of signals coming from different sensory channels. The "interpersonal self" is the second lowest level and allows to control personal behavior during social interactions. These two representations of the self could be linked to what Jeannerod (2003) characterized as self-recognition (i.e. recognizing oneself as the owner of a body (ownership) and the agent of actions (agency)) which supposedly arise from proprioceptive and sensory body signals, and the internal representation of motor intentions. From Neisser's perspective (1995), three other levels should be considered. By the fourth year of life the "temporally extended self" appears, a representation of our own life story as we know it. This could be linked to autobiographical memory even if it also encompasses a representation of the future life story. The "private self" reflects knowledge that our conscious experience as well as our thoughts exclusively own and is not shared with other agents. Finally, the "conceptual self" represents the sociocultural experience based on cultural verbal information. If the "private self' can be linked to mental states ascription abilities, a topic of major interest in the field of social cognitive neuroscience "the conceptual self" has clearly received less attention in the field. More recently and based on the various operationalizations of the self in the neuroscientific literature, a practical distinction between physical and psychological aspects of the self has been proposed (Gillihan \& Farah, 2005; see figure 2.A. for a schematic representation of the correspondences between the social psychological and the neuroscientific conceptions). The physical aspects of the self would embrace the awareness of our body (e.g. Devinsky, 2000) and self-body recognition skills (e.g. Keenan, Freund, Hamilton, Ganis, \& Pascual-Leone, 2000), whereas psychological aspects of the self would rather include autobiographical memory and the 
conscious experience of first-person perspective (or "egocentric frame of reference", Vogeley and Fink, 2003). The latter could be linked to the "private self" described earlier, as it corresponds to the representation of information available to the self (e.g. beliefs, perceptions) as opposed to others. Finally, the feeling of agency would constitute a bridge between the physical and psychological aspects of the self as it relies on both a recognition of one's body as one's own and of the actions as caused by oneself.

\section{// Insert Figure 2 around here //}

As illustrated in figure 2, important overlaps exist between the taxonomy of the self as described from the social psychological and the neuroscientific point of view. We will distinguish three levels that partly overlap with the existing distinctions from social psychology and neuroscience, namely the perceptual level, the action level and the mental-state level. At the perceptual level, we will discuss the human ability to identify his/her own body and to distinguish it from the body of others. At the action level, we will discuss the human ability to experience agency for self-generated motor actions (opposed to others' actions) as well as the control of imitation (i.e. distinguish between self-generated and other-generated motor representations). Finally, at the mental-state level, we will discuss the human's ability to distinguish between his/her own mental states and those of other agents. Importantly, most experimental tasks that are related to self-other distinction on these three levels are based on congruency manipulations, where the self and the other perspective are in conflict. In such situations, it is necessary to distinguish self- and other-related representations to avoid confusion. 


\section{Self-other distinction at the perceptual level}

Our body seems to be unambiguously related to ourselves and it seems there is not much room for confusing it with others. However, the neuropsychological literature (e.g. Brugger \& Lenggenhager, 2014) and experimental findings (e.g. Petkova \& Ehrsson, 2008) suggest that selfother distinction is also required on this level. We will here discuss evidence originating from experimental manipulation and psychopathological disorders that alter the sense of ownership. This should allow us to gain a better understanding of the neural structures supporting the cognitive mechanisms in play to identify other individuals and to distinguish other bodies or limbs from our own.

From an experimental point of view, the most common paradigm known to interact with the cognitive processes supporting the distinction between self and others' body parts is the "rubber hand illusion" (RHI, Braun et al., 2018; Botvinick \& Cohen, 1998), or its virtual reality version, the "virtual hand illusion" (VHI). In this paradigm, an artificial hand is placed in front of a participant, while his/her own hand is hidden from view. The experimenter then repeatedly strokes both the artificial hand and the real hand in synchrony. In most participants, this induces a feeling of ownership over the artificial hand that can be quantified through proprioceptive drifts when having to localize the actual location of their hand (e.g. Tsakiris \& Haggard, 2005), emotional reactions towards the artificial hand (e.g. Ehrsson, Wiech, Weiskopf, Dolan \&Passingham, 2007) or even explicit statements of participants (e.g. Botvinick \& Cohen, 1998). A few neuroimaging studies used this paradigm to reveal the brain regions associated with experimentally induced sense of ownership over other's body part. In this context, numerous 
brain structures, spread among the frontal, parietal, occipital and limbic cortex, have been identified to play a role (see Braun et al., 2018, for a review). Interestingly, among the parietal regions identified, the supramarginal gyrus (SMG) which constitutes a part of the dorsal TPJ, is one of the most consistently reported structures (e.g. Brozzoli, Gentile \& Ehrsson, 2012; Ehrsson, Spence \& Passingham, 2004; Gentile, Guterstam, Brozzoli \& Ehrsson, 2013). The activity of the SMG has however been found to positively correlate with the size of the proprioceptive drift (Brozzoli, Gentile \& Ehrsson, 2012), which indexes the experience of ownership over the artificial hand. It should be noted that RHI paradigms however do not require to actively distinguish between self and other representations. At this point, this correlation remains then difficult to interpret. Moreover, one could also consider that participants actually need to distinguish their hand from the other one, only when experiencing induced ownership over the rubber hand given to its explicit artificial nature.

If integrating others' representations may be useful for social interactions (Gallotti \& Frith, 2013), being able to distinguish other limbs from our owns remains of critical importance from an emotional point of view. In the absence of such ability, any stimulation applied to other bodies would generate congruent perceptions or sensations in the observer and other oriented threats would trigger robust emotional response in the absence of actual danger. Accurate self-other distinction is then crucial to avoid the occurrence of inappropriate personal distress as well as to prevent our own state to interfere with the understanding of others' physical experiences (Lamm, Bukowski \& Silani, 2016). Some recent studies investigated self-other distinction at the emotional level in the context of perceptual stimulations. Classically, in these experiments two participants simultaneously underwent an affective touch of incongruent valence and are required 
to judge the experienced pleasantness or unpleasantness of stimulation for themselves or for their partner (Silani, Lamm, Ruff \& Singer, 2013; Tomova, von Dawans, Heinrichs, Silani \& Lamm, 2014). It has been found that the valence of one's own affective touch slightly biased judgements of the affect touch displayed to partner in an egocentric way (Silani et al., 2013). Critically for the current issue, the authors observed that temporarily disruption of the right SMG (dorsal anterior TPJ, in our framework) substantially increase this bias, directly interfering with the participant's ability to distinguish between their perceptual experience and those of others. Overall, these findings corroborate the role of the SMG for self-other processing at the perceptual level. However, whether these findings support self-other merging processes, self-other distinction processes, or both kind of phenomena by merely allowing us to shift between representations remains unclear.

While the "rubber hand illusion" would imply cognitive mechanisms dealing between self and others' representations, by inducing in participants the feeling of ownership over others' limbs, the reversed psychological states (i.e. feeling distance with our own body), has been reported in different psychopathological and neurological populations. This is for example the case in Out-of-body experience, somato-paraphrenia and depersonalization. Patients reporting Out-of-body experiences typically describe episodes in which their "self" were located outside of their physical body, literally seeing their body from another point of view (but still identifying it as their body). Somato-paraphrenia is a delusional state in which patients deny ownership over one (or more) of their paralyzed limbs, often associated with the tendency to attribute it to someone else, even if provided with undeniable proof that the limb in question belongs to them. Finally, depersonalization is a psychological condition characterized by a persistent feeling of 
detachment from one's own self and body, for which patients classically report the sensation of being an outside observer (but without losing any contact with reality). Interestingly, and congruently with the experimental findings described above, these distinct clinical syndromes all seem to rely partly on the dorsal part of the TPJ. Lesion overlap analysis of patients reporting out-of-body experiment point to a crucial role of the TPJ (Blanke \& Arzy, 2005). Confirming this clinical evidence, it has been experimentally shown that stimulation of the brain's right angular gyrus (dorsal posterior TPJ, in our framework) can elicit out-of-body experiences (Blanke, Ortigue, Landis \& Seeck, 2002). Historically, a variety of "self-awareness" disorders, including somato-paraphrenia have also been associated to lesions of the TPJ (Berlucchi \& Aglioti, 1997). Recent integrative works however support the involvement of a much more extended network (Gandola et al., 2012). To our knowledge, the neural bases associated with depersonalization remain to date largely unknown but existing models seem to not attribute a particular role to the TPJ (Sierra, 2009; Sierra \& Berrios, 1998).

Overall, the role of the TPJ in self-other distinction at the perceptual level remains relatively unclear. While there is evidence of TPJ involvement in paradigms such as the rubber hand illusion, how this activation relates to self-other distinction is not completely clear and is probably related to task demands. The findings suggest that the dorsal part of the TPJ (SMG and AG) might be involved in conditions implying active self-processing and multisensory integration of body-related information. Supporting this interpretation, clinical data support a link between the dorsal part of the TPJ and transient episodes of self-awareness processes, but not of more permanent alteration. At this stage, the small number of experimental studies directly 
investigating self-other distinction at the perceptual level prevents any strong conclusion, and future works is needed to fully understand the role of the TPJ in relation to this ability.

\section{Self-other distinction at the action level}

In the following, we will discuss evidence concerning the role of the TPJ in self-other distinction related to motor control. Distinguishing between self and other-generated motor actions refers to the sense of agency, which has been described as 'the sense that I am the one who is causing or generating an action' (Gallagher, 2000). Experiencing the sensory consequences of an action as being caused by one's own behavior is crucial for interacting with the social and non-social environment. It represents a key element during joint action as it allows to evaluate one's own contribution to the joint action outcome (Jeannerod, 2003).

When studying the sense of agency, as explicitly experienced, participants are usually asked to perform a motor task for which they see a feedback of the motor behavior on a screen. The feedback could depict either their own movements, their own altered movements (e.g. rotated by $25^{\circ}$ ), or the movements of someone/something else (e.g., a confederate or computer program, Braun et al., 2018). Sometimes, the movement displayed is jointly controlled by both the participants and the confederate (e.g. Wegner \& Whealthy, 1999). In all the cases, participants are then asked to judge whose movement is seen on the screen. Neuroimaging studies, relying on such paradigms, identified different brain regions to be associated with the sense of agency. Initial works suggested an involvement of the anterior insula and the angular gyrus (AG), a dorsal part of the TPJ, specifically in the right hemisphere (e.g. Farrer \& Frith, 
2002; Farrer et al., 2003). Critically for the current issue, the highest TPJ activations were observed in conditions in which participants experienced minimal control over others' movements (Farrer et al., 2003; see also Spengler, von Cramon, \& Brass, 2009, illustrated in Figure 3). More recent findings also included the supplementary motor area, the ventral premotor cortex, the dorsolateral prefrontal cortex and the cerebellum (David, Newen \& Vogeley, 2008; Haggard, 2017). However, the specific role of these different regions to the emergence the sense of agency is still poorly understood. This important number of brain regions found to be linked with agency may be the consequence of the integration of literatures focusing on distinct cognitive phenomena (Haggard, 2017). In addition to the explicit measures of agency described above, a well-studied psychological phenomenon, the 'intentional binding' effect, is often considered as a potential implicit marker of agency (Braun et al., 2018). Intentional binding describes the observation that people underestimate the time interval between an action (e.g. pressing a key) and the consequence of that action (e.g. a tone), compared with a similar situation where the behavior is not self-initiated but externally induced. Interestingly, in one neuroimaging study that reports behavioral evidence of "intentional binding", the authors found no correlation between the angular gyrus and the temporal measure of sense of agency (Kühn, Brass \& Haggard, 2013). In contrast, they found that the supplementary motor area (SMA) was more strongly associated with the perceived action-tone interval than with perception of a control interval following a passive movement. This suggest that different brain structures would support the explicit feeling of agency and its implicit components. The existence of a link between explicit and implicit measures of the sense of agency is however controversial (Dewey \& 
Knoblich, 2014) and some authors argue that temporal binding is not related to agency but rather reflects a more general principle of causal perception (e.g. Buehner, 2012).

// Insert Figure 3 around here //

While experiencing agency requires to determine whether the sensory consequences of actions are self-caused or not, the control of imitative response tendencies require the ability to distinguish conflicting motor representations prior to their execution (Sowden \& Catmur, 2015). It has been argued that action observation leads to an activation of an internal motor representation in the observer (Brass \& Heyes, 2005). If this externally triggered response tendency is incongruent with one's own motor intention, self-other distinction is required (Brass et al., 2009) to distinguish one's own motor intention from the externally triggered motor intention. Most social interactions require the production of complementary actions (e.g. if someone open the door in front of me, I should be primed to enter it and not open it a second time). As consequence, indiscriminate imitation is not an appropriate by default response and it is then of critical importance to distinguish between self-generated motor representations and the ones activated by the observation of others' actions (Brass, Zysset \& von Cramon, 2001). Concerning automatic imitation, there is much less variability among the paradigms in use. Classically (e.g. Brass, Bekkering, Wohlschläger \& Prinz, 2000), participants are instructed to lift their index finger in response to a particular stimulus (e.g. the number " 1 ") and their middle finger in response to a second one (e.g. "2"). At the same time, a hand on the screen also lift its index finger or its middle finger, producing then either congruent conditions (in which the hand on the screen activates motor representations congruent with the movement that participants have 
to produce) and incongruent conditions (in which the hand on the screen activates motor representations that would interfere with the movement that participants have to produce and that should then be inhibited). Initial neuroimaging experiment relying on such paradigms identified the anterior fronto-medial cortex (aFMC, Brass et al. 2001; 2005; 2009) and the TPJ (Brass et al. 2005; 2009; Marsh, Bird \& Catmur, 2016) as the brain substrate for the control of automatic imitative response tendencies. The brain network associated with the control of automatic imitation has however been questioned in a recent meta-analysis (Darda \& Ramsey, 2019). These authors reported consistent evidence supporting that the control of automatic imitation is guided by brain regions associated with domain-general abilities allowing to deal with multiple demands (e.g. dorsolateral frontoparietal cortex), relativizing the role of the TPJ. Clearly, further imaging work is needed to resolve the inconsistencies between older studies on the inhibition of imitative response tendencies and more recent work. Going further than these neuro-imaging studies, different authors directly tackled the involvement of the TPJ using transcranial stimulation techniques (Bardi, Gheza \& Brass, 2017; Hogeveen, Obhi, Banissy, Santiesteban, Press, Catmur \& Bird, 2014; Santiesteban, Banissy, Catmur \& Bird, 2015; Sowden \& Catmur, 2015). All these studies supported the role of the TPJ in the control of automatic response tendencies. Some experiments suggest that the TPJ is specifically responsible for switching between self and other representations as a domain general process (Hogeveen et al., 2014; Sowden \& Catmur, 2015) while the frontal regions would be more sensitive to task demands (Hogeveen et al., 2014) and may then reflect a more explicit control process.

Overall, various findings support an active role of the TPJ in self-other distinction at the action level. Precisely, the TPJ has been found to be linked to the experience of agency as well as 
to the control of imitation tendencies. In the case of the sense of agency, even if the role of the TPJ is relatively well supported for explicit measures, it currently exists no evidence supporting its involvement for implicit measures. Furthermore, it has been proposed that the angular gyrus (dorsal posterior TPJ, in our framework) might provide a subjective marker of volition (Chambon, Wenke, Fleming, Prinz \& Haggard, 2013) while other structures (e.g. SMA) may support more implicit aspects of sense of agency (Kühn et al., 2013). Congruently with the conclusion of the previous section, the dorsal parts of the TPJ have been identified to play a role in self-other distinction at the action level. In both sections, activation of the TPJ is reported when the participants experience a conflict between representations (e.g. "I explicitly know that this hand is not mine while I am prompted to think so", "the movement that I'm seeing is not congruent with the one I produced"). The TPJ then seems to be responsible for the control of shared representations, allowing to discriminate between self-related and others-related representations. An alternative hypothesis would be that activation in the TPJ reflects the detection of a conflict between multiple representations. Both hypotheses would be congruent with the evidence reported so far. The studies of Silani et al. (2013) focusing on self-other distinction at the emotional level would however be more consistent with the first hypothesis. The authors reported higher activity in right SMG in incongruent (conflict) as compared with congruent (no conflict) condition when participants had to make empathic judgments about another persons' affective states only, and not when engaged in self-judgments.

\section{Self-other distinction at the mental-state level}


Self-other distinction at the mental-state level has received a lot of attention, following long traditions of research in clinical psychology (Rogers, 1951), developmental science (Piaget \& Inhelder, 1948) and ethology (Premack \& Woodruff, 1978). Not only representing others' emotions, intentions, beliefs or even percepts, but being able to distinguish them from our own psychological states provide a clear benefit for individuals of both social and non-social species (Hecht, Patterson \& Barbey, 2012). Multiple terms have been used through disciplines and times to describe the ability to represent other's mental states while being able to distinguish them from our own (see Quesque \& Rossetti, in press). Because we do not want to enter the discussion about terminology in the current article, we will use the neutral and unspecific term "self-other distinction at the mental-state level". In this last section, we aim to discuss the role of the TPJ for self-other distinction at the mental-state level, based on recent experimental contributions, as well as the results of meta-analyses we conducted using Neurosynth (Yarkoni, Poldrack, Nichols, Van Essen \& Wager, 2011).

Contrasting with the two previous sections, the large number of neuroimaging studies available allowed us to conduct reliable 'association test' (Poldrack, 2006; 2011). This consists in evaluating how selectively an activation in a particular brain region is linked to specific cognitive processes (displaying voxels that are reported more often in articles that include a specific term than in articles that do not). We utilized the functional (f)MRI meta-analytic resource Neurosynth (http://neurosynth.org, 14371 total studies in spring 2019, Yarkoni et al., 2011) to run this test and thus identify brain regions implicated for self-other distinction at the mental-state level. Specifically, the specific terms we used to determine the inclusion of studies are 'Mental states' ( $n=141$ studies, references of all studies included are available as supplementary data), 
'Mentalizing' ( $\mathrm{n}=151$ studies, $14.5 \%$ of overlap with studies included for 'Mental states'), and 'Theory of Mind' ( $\mathrm{n}=181$ studies, respectively $28.7 \%$ and $9.4 \%$ of overlap with studies included for 'Mental states' and 'Mentalizing'). One might argue that using search terms is not a good proxy to identify specific cognitive processes and may lead to scattered activation maps, integrating data from distinct literatures. On the contrary, activation maps obtained with such wide inclusion criteria present the advantage of limiting the influence of specific experimental demands in favor of the common cognitive process implied in all studies included (Yarkoni et al., 2011). In the present case, the chosen terms remain quite specific for a specific domain of research. As a matter of fact, we can see in Figure 3 that the brain regions selectively associated with the three terms we used are extremely similar. Additionally, no important differences seem to exist between the left and right hemisphere. The brain network identified here as responsible for self-other distinction at the mental-state level would then bilaterally include the medial prefrontal cortex (mPFC), the posterior part of the TPJ (AG and pSTS) and the most rostral portions of both the STG and the middle temporal gyrus (MTG). Finally, the inferior frontal gyrus (IFG) could also be mentioned. This last structure in however not found in the classical brain activation pattern observed for "Mentalizing" (contrasting with "Mental states" and "Theory of Mind"). Considering that "Mentalizing" and "Theory of Mind" are widely used as synonyms (Quesque \& Rossetti, in press) one should have expected to identify a common brain network for the two terms. The specific absence of activation of the IFG for studies on "Mentalizing" then suggests than its role would be more dependent of typical experimental demands regarding to specific literatures than to the general ability to distinguish self-and other mental states. 


\section{// Insert Figure 4 around here //}

The neural network identified here as being associated with the ability to distinguish self from other's mental states is highly congruent with the one that has been historically reported when focusing on the ability to represent others' false beliefs (e.g. "I know that they will search for them ball at the wrong location, if I moved it while they were away", Wimmer \& Perner, 1983), and which encompasses fronto-medial and temporo-parietal regions (Gallagher et al., 2000; Saxe \& Kanwisher, 2003; Vogeley et al., 2001). Interestingly, implicit version of false beliefs tasks, in which participants are not specifically instructed to represent others' beliefs, also revealed the involvement of the more dorsal parts of the TPJ (see Boccadoro et al., 2019 for a mega-analytics approach pooling data from multiple fRMI studies). Whether the explicit and implicit versions of false-beliefs tasks address a common, or different underlying, cognitive process(es) is however still debated (Apperly \& Butterfill, 2009; Carruthers, 2015; Heyes, 2014).

As false-beliefs tests represent the most used task to investigate self-other distinction at the mental-state level, one may however wonder to what extent the brain structures identified here, and particularly the TPJ, could also support the ability to represent, and distinguish from our own, others' types of mental states such as emotions or perceptions (Saxe \& Wexler, 2005). This question appears to be especially complex as most of the tasks in use to evaluate these abilities vary in many ways in addition to the specific types of mental states involved (e.g. relying on "narratives" or "animations" for beliefs, vs "pictures of expressive faces" for emotions, vs "videos of moving eyes" or "drawing of a scene implying another agent" for perceptual inferences). In order to overcome such limits, Zaitchik et al. (2010) used a highly standardized 
paradigm relying in a within-subject design, a single type of stimulus (verbal information), a single type of psychological process (sentence comprehension), identical stimuli (except for the mental state to be attributed), and compared the brain activations for the attribution of beliefs, emotions, and perceptions to other agents. They found that all types of mental states inferences involve the TPJ. However, they identified that the attribution of beliefs more strongly recruited both the posterior STS and the inferior parietal lobule (IPL, which includes the SMG and AG) than did emotions or perceptions. Partially responding to the same question, some authors (Schurz, Aichhorn, Martin \& Perner, 2013) systematically compared, across studies, the brain areas engaged in false belief reasoning and in visual perspective-taking (e.g. represent that "from their point of view the 6 is seen as a 9", Quesque, Chabanat \& Rossetti, 2018). They reported common activation for both tasks in the dorsal part of the left (but not right) TPJ, suggesting, in line with the previous study, that the TPJ might be responsible for representing different perspectives in a domain general fashion. More recent studies (Schurz, Kronbichler, Weissengruber, Surtees, Samson \& Perner, 2015) moreover demonstrated that this supposed domain general role of the TPJ to represent different perspectives is limited to social inferences (i.e. "when having to consider the perspective of another agent" vs "the perspective of an arrow”). Finally, the same research team (Arora, Schurz \& Perner, 2017) compared the brain areas engaged in visual perspective-taking with numerous other social cognition tasks. Again, they identified overlap between brain activations for visual perspective-taking and other types of mental state attribution. The left TPJ, however, seemed to selectively respond to tasks that require to consider a de-centered perspective (as it is the case "false belief" and "visual perspective- 
taking" tasks), and not to tasks which can be completed by the discrimination of physical features (e.g. categorizing emotional faces).

The involvement of the TPJ in self-other distinction on the mental-state level has been suggested almost 20 years ago (Gallagher et al., 2000). Numerous studies have now confirmed this initial finding and the meta-analyses that we conducted here led to the identification of a quite well-defined brain network for the ascription of mental states to others, including the TPJ. Altogether, the findings discussed in the present section suggest that the TPJ could be responsible for self-other distinction in a domain-general manner, independently of the content of the representation. Finally, differences between left and right TPJ have been consistently found (e.g. Aichhorn et al., 2006, in favor of the ITPJ; Saxe \& Kanwisher, 2000, in favor of the rTPJ). In contrast to this, our current meta-analysis does not reveal important differences between hemispheres. Whether this inconsistency can be explained by variations over relatively small samples in neuroimage studies or correspond to a lack of specificity in our current analyses remains to be further tested.

\section{The TPJ as a domain general neural correlate of self-other distinction}

Here, we discussed the role of the TPJ in self-other distinction for the different levels of “self" representation. At the perceptual level, we reported that the involvement of the TPJ is probably limited to conditions implying active and transient self-other distinction processing episodes and multisensory integration of body-related information. At the action level, the TPJ seems to be play a role in the control of imitation as well as in the explicit, but not implicit, sense of agency. Finally, at the mental-state level, the implication of the TPJ in self-other distinction is 
now well validated for different type of representations (beliefs, visual perspectives, etc). Altogether, these findings suggest that the TPJ may be the neural substrate responsible of domain-general transient form of self-other distinction (opposed to enduring state), as it has been proposed by Van Overwalle (2009), and may interact with different types of representations depending of the type of psychological processes involved. Two concurrent hypotheses concerning the specific role of the TPJ have been proposed in this manuscript. The TPJ could be responsible for conscious forms of control over self and other representations. It would allow to distinguish between representations, and consequently contribute to inhibit the influence of the non-relevant one. Alternatively, the TPJ may simply be activated when experiencing incongruencies between self and other representations and then constitutes a signal of the conflict.

Supporting both interpretations, it appears that the TPJ is not implied in all types of social judgements but mostly in those that will require to distinguish between our actual experience of the world and the one of another person. Precisely, we reported activation of the TPJ in explicit and implicit self-other judgement tasks, but only in conditions in which there was a discrepancy between self and other's representations (e.g. in the case of representation of "false beliefs"). Activation of the TPJ in case of congruent self and other's representations, when the task explicitly requires participants to represent both representations (e.g. in the case of representation of others" "true beliefs"), however constitute a strong argument against the signal of conflict hypothesis. Moreover, if, according to the second hypothesis, the TPJ would merely be a tracker of conflict between mental representations, it remains to understand which other brain structure allows to elaborate self -other distinctions in the cases of mismatching between representations. 
Different options (e.g. amplify either self or other's representations depending on task demands, inhibiting interfering representations, etc.) have been identified (Lamm et al., 2016). The fact that temporarily disruption of the TPJ however impede the ability to distinguish between perspectives, but not with the interferences between perspectives (Silani et al., 2013), would also encourage us to support the first interpretation. Even if future work directly aiming at comparing both hypotheses would be needed to draw firm conclusions, this motivates us to presently favor the view of the TPJ as responsible of domain-general transient form of self-other distinction.

Despite the lack of a general architecture among social cognitive processes (Happé et al., 2017; Quesque \& Rossetti, in press), some authors suggested the existence of a form of hierarchy between the control of shared representations at the action level and the attribution of mental states to others (e.g. Brass et al., 2009; Bardi \& Brass, 2016), already pointing the potential role of the TPJ. We here encompass this account and extend it to the perceptual level. Early in development, a shared representation system (not differencing between agents) would arise from repeated exposure to associations between a motor command and its perceptual consequences (see Brass \& Heyes, 2005). With experience, this system dissociates between events that are contingent with one's actions and those that are not, resulting in the development of a sense of the self (and of others). Finally, this ability to co-represent and switch between self-related and other-related representations would constitute the pre-condition to all the socio-cognitive mechanisms discussed in the present manuscript, at the perceptual, motor, but also mental-state levels. From an ontogenetic view, this account is extremely parsimonious. The TPJ, supporting multisensory integration, would constitute a privileged structure for anchoring the self in private sensory information. Through social interaction, higher-order cognitive mechanisms such as the 
ability to represent others' beliefs might develop from such low-level mechanisms (see Heyes, 2018).

On the basis of the present review, one could expect to generalize such hierarchy to other cognitive processes. Considering that the TPJ is responsible for the distinction between self and other (i.e. "the control of shared representations") in a domain general manner, it may then be implied in all mental activities that require to shift from a representation to another (see also Sowden \& Catmur, 2015 for a similar view limited to social cognition). This is of course the case of many social processes, going from the identification of the ownership of body parts to the attributions of mental states, but it may also happen in other (non-social) cognitive operations. Comforting our current suggestion, the TPJ has been identified as playing a role in various mental operations requiring to shift between representations, as for example thinking about our own thought (Lombardo et al., 2010), thinking of future events (Abraham, Schubotz \& von Cramon, 2008), considering the perspective of one's future self (Soutschek, Ruff, Strombach, Kalenscher \& Tobler, 2016), or interpreting ambiguous sentences that require to represent different perspectives (Van Ackeren, Casasanto, Bekkering, Hagoort \& Rueschemeyer, 2012).

In line the present account, Mitchell (2007) suggested that activity in the temporo-parietal junction would not be selective for mental-states ascription but would rather reveal a general switch in attention. A similar idea has also been proposed by Corbetta et al. (2008). According to them, high-level cognitive abilities such as mental states attribution (requiring to shift between mental representations) would be the mere abstraction of a low-level, and evolutionary inherited, ability to change a current course of action in favor of the production of another (reorienting 
attention accordingly). Whether the general ability to switch between self and other representations described here as a core function of the TPJ could be extended to attentional responses remains for the moment an open question and should be addressed in future contributions. Interestingly, many authors have however underlined the involvement of the TPJ for the reorientation of attention as well as for the ability to represent another mental states (Corbetta et al., 2008; Decety \& Lamm, 2007; Young et al., 2010). Supporting an alternative view, some experimental work identified a reliable anatomical distinction within the TPJ between the activations linked to attentional responses and the activations linked to social judgements (Krall et al., 2013; Silani et al. 2013). In the same vein, Mars et al. (2012) reported distinct anatomical connectivity profiles within the different sub-parts of the TPJ. It would be possible then that attentional reorientation and the control of self and other representations are relying on neighboring but distinct regions within the TPJ and constitute different cognitive processes.

Even if limiting the role of the TPJ to the control of self and other representations, it remains to determine if the same regions within the TPJ support all types of self-other distinctions. In the past, some authors argued that different regions of the TPJ are involved for distinct types of social judgements (Carter \& Huettel, 2013). Here, most of the evidence we reviewed suggest than the IPL would be responsible for a domain general ability to switch between representations. At the perceptual level, both the SMG and the AG have been identified to contribute to distinguish between self and other perspectives. At the action level, the AG is classically considered as a subjective marker of volition. Finally, at the mental-state level, as mentioned earlier, there is an important lack of specificity as most of the time the TPJ is considered as a whole. The present meta-analyses would however invite to link mental states 
ascription processes to the most posterior and dorsal part of the TPJ (AG). This account corroborates previous works which directly aimed at comparing the neural substrate of different types of social cognition tasks (Arora et al., 2017; Mar, 2011; Perner \& Leekam, 2008).

Precisely, the authors concluded that the processing of perspective differences relies on the dorsal parts of the TPJ (SMG, AG) while more ventral parts (STG, STS) would also be involved in social cognition processes, but which do not involve to distinguish between self-and others representations (e.g. categorizing others' emotions from their faces or kinematics).

\section{Conclusion and perspectives}

Altogether, the findings discussed in the present paper suggest that the TPJ may be responsible for control over self and other representations, in a domain general manner. Past work suggested that the TPJ may constitutes the common neural substrate for all types of mental states ascription to others (Zaitchik et al., 2010). Adopting a neural recycling approach, some authors (Bardi \& Brass, 2016) postulated that a shared neural network would support high-level social judgements about others' internal states and the control of shared representation at the motor level. We here encompass this account and extend it to all levels of self-other representations, offering a general architecture for the emergence of numerous socio-cognitive processes. From a neural point of view, we defend that this ability to enhance task-relevant representations in case of mismatches between self-related and other-related representations would most probably rely on the dorsal part of the TPJ (SMG, AG). Finally, we would recommend researchers to be as specific as possible when referring to brain regions within the TPJ, when it is possible. Precisely, we encourage to systematically refer to the precise anatomical 
structures in play rather than the abstract concept of "TPJ". Adopting this recommendation would presumably contribute to a better understanding of the neural substrate supporting the ability to distinguish between self and other representations. 


\section{References}

Abraham, A., Schubotz, R. I., \& von Cramon, D. Y. (2008). Thinking about the future versus the past in personal and non-personal contexts. Brain research, 1233, 106-119.

Aichhorn, M., Perner, J., Kronbichler, M., Staffen, W., \& Ladurner, G. (2006). Do visual perspective tasks need theory of mind?. Neuroimage, 30(3), 1059-1068.

Apperly, I.A., \& Butterfill, S.A. (2009). Do humans have two systems to track beliefs and belief-like states? Psychological Review. 116, 4, 953-70.

Arora, A., Schurz, M., \& Perner, J. (2017). Systematic comparison of brain imaging metaanalyses of ToM with vPT. BioMed research international.

Arzy, S., Thut, G., Mohr, C., Michel, C. M., \& Blanke, O. (2006). Neural basis of embodiment: distinct contributions of temporoparietal junction and extrastriate body area. Journal of Neuroscience, 26(31), 8074-8081.

Bardi, L., \& Brass, M. (2016). The control of shared representations and social cognition. Shared representations. Cambridge University Press, Cambridge, 151-170.

Bardi, L., Gheza, D., \& Brass, M. (2017). TPJ-M1 interaction in the control of shared representations: New insights from tDCS and TMS combined. NeuroImage, 146, 734-740.

Berlucchi, G., \& Aglioti, S. (1997). The body in the brain: neural bases of corporeal awareness. Trends in neurosciences, 20(12), 560-564. 
Blakemore, S. J., Smith, J., Steel, R., Johnstone, E. C., \& Frith, C. D. (2000). The perception of self-produced sensory stimuli in patients with auditory hallucinations and passivity experiences: evidence for a breakdown in self-monitoring. Psychological medicine, 30(5), 11311139.

Blanke, O., \& Arzy, S. (2005). The out-of-body experience: disturbed self-processing at the temporo-parietal junction. The Neuroscientist, 11(1), 16-24.

Blanke, O., Ortigue, S., Landis, T., \& Seeck, M. (2002). Neuropsychology: stimulating illusory own-body perceptions. Nature, 419(6904), 269.

Boccadoro, S., Cracco, E., Hudson, A., Bardi, L., Nijhof, A., Wiersema, J. R., ... \& Mueller, S. (2019). Defining the neural correlates of spontaneous theory of mind (ToM): An fMRI mega-analytic investigation. bioRxiv, 560953.

Bogen, J. E., \& Bogen, G. M. (1976). Wernicke's region-where is it?. Annals of the New York Academy of Sciences, 280(1), 834-843.

Botvinick, M., \& Cohen, J. (1998). Rubber hands 'feel'touch that eyes see. Nature, 391(6669), 756.

Braun, N., Debener, S., Spychala, N., Bongartz, E., Sorös, P., Müller, H. H. O., \& Philipsen, A. (2018). The senses of agency and ownership: a review. Frontiers in psychology, 9, 535. 
Brass, M., Bekkering, H., Wohlschläger, A., \& Prinz, W. (2000). Compatibility between observed and executed finger movements: Comparing symbolic, spatial, and imitative cues. Brain and Cognition, 44, 124-143.

Brass, M., Derrfuss, J., \& von Cramon, D. Y. (2005). The inhibition of imitative and overlearned responses: a functional double dissociation. Neuropsychologia, 43(1), 89-98.

Brass, M., \& Heyes, C. (2005). Imitation: is cognitive neuroscience solving the correspondence problem?. Trends in cognitive sciences, 9(10), 489-495.

Brass, M., Ruby, P., \& Spengler, S. (2009). Inhibition of imitative behaviour and social cognition. Philosophical Transactions of the Royal Society B: Biological Sciences, 364(1528), 2359-2367.

Brass, M., Zysset, S., \& von Cramon, D. Y. (2001). The inhibition of imitative response tendencies. Neuroimage, 14(6), 1416-1423.

Brozzoli, C., Gentile, G., \& Ehrsson, H. H. (2012). That's near my hand! Parietal and premotor coding of hand-centered space contributes to localization and self-attribution of the hand. Journal of Neuroscience, 32(42), 14573-14582.

Brugger, P., \& Lenggenhager, B. (2014). The bodily self and its disorders: neurological, psychological and social aspects. Current opinion in neurology, 27(6), 644-652.

Buehner, M. J. (2012). Understanding the past, predicting the future: causation, not intentional action, is the root of temporal binding. Psychological science, 23(12), 1490-1497. 
Bukowski, H., \& Lamm, C. (2017). Temporoparietal Junction. In V. Zeigler-Hill \& T. K. Shackelford (Eds.), Encyclopedia of Personality and Individual Differences (pp. 1-5). New York, NY: Springer. doi:10.1007/978-3-319-28099-8_863-1

Bzdok, D., Langner, R., Schilbach, L., Jakobs, O., Roski, C., Caspers, S., ... Eickhoff, S. B. (2013). Characterization of the temporo-parietal junction by combining data-driven parcellation, complementary connectivity analyses, and functional decoding. NeuroImage, 81, 381-392.

Cabeza, R., Ciaramelli, E., \& Moscovitch, M. (2012). Cognitive contributions of the ventral parietal cortex: an integrative theoretical account. Trends in cognitive sciences, 16(6), 338-352.

Carruthers, P. (2015). Mindreading in adults: Evaluating two-systems views. Synthese, 116.

Carter, R. M., \& Huettel, S. A. (2013). A nexus model of the temporal-parietal junction. Trends in cognitive sciences, 17(7), 328-336.

Chambon, V., Wenke, D., Fleming, S. M., Prinz, W., \& Haggard, P. (2012). An online neural substrate for sense of agency. Cerebral Cortex, 23(5), 1031-1037.

Corbetta, M., Patel, G., \& Shulman, G. L. (2008). The reorienting system of the human brain: from environment to theory of mind. Neuron, 58(3), 306-324

Corbetta, M., \& Shulman, G. L. (2002). Control of goal-directed and stimulus-driven attention in the brain. Nature reviews neuroscience, 3(3), 201. 
Darda, K. M., \& Ramsey, R. (2019). The inhibition of automatic imitation: a meta-analysis and synthesis of fMRI studies. NeuroImage, 197, 320-329.

Daprati, E., Franck, N., Georgieff, N., Proust, J., Pacherie, E., Dalery, J., \& Jeannerod, M. (1997). Looking for the agent: an investigation into consciousness of action and selfconsciousness in schizophrenic patients. Cognition, 65(1), 71-86.

David, N., Newen, A., \& Vogeley, K. (2008). The "sense of agency" and its underlying cognitive and neural mechanisms. Consciousness and cognition, 17(2), 523-534.

Decety, J., \& Lamm, C. (2007). The role of the right temporoparietal junction in social interaction: how low-level computational processes contribute to meta-cognition. The Neuroscientist, 13(6), 580-593.

Devinsky, O. (2000). Right cerebral hemisphere dominance for a sense of corporeal and emotional self. Epilepsy and Behavior, 1, 60-73.

Dewey, J. A., \& Knoblich, G. (2014). Do implicit and explicit measures of the sense of agency measure the same thing?. PloS one, 9(10), e110118.

Ehrsson, H. H., Spence, C., \& Passingham, R. E. (2004). That's my hand! Activity in premotor cortex reflects feeling of ownership of a limb. Science, 305(5685), 875-877.

Ehrsson, H. H., Wiech, K., Weiskopf, N., Dolan, R. J., \& Passingham, R. E. (2007). Threatening a rubber hand that you feel is yours elicits a cortical anxiety response. Proceedings of the National Academy of Sciences, 104(23), 9828-9833. 
Farrer, C., Franck, N., Georgieff, N., Frith, C. D., Decety, J., \& Jeannerod, M. (2003). Modulating the experience of agency: a positron emission tomography study. Neuroimage, 18(2), 324-333.

Farrer, C., \& Frith, C. D. (2002). Experiencing oneself vs another person as being the cause of an action: the neural correlates of the experience of agency. Neuroimage, 15(3), 596-603.

Gallagher, H. L., Happé, F., Brunswick, N., Fletcher, P. C., Frith, U., \& Frith, C. D. (2000). Reading the mind in cartoons and stories: an fMRI study of 'theory of mind'in verbal and nonverbal tasks. Neuropsychologia, 38(1), 11-21.

Gallagher, S. 2000a. Philosophical conceptions of the self: implications for cognitive science. Trends in Cognitive Science 4 (1): 14-21.

Gallotti, M., \& Frith, C. D. (2013). Social cognition in the we-mode. Trends in cognitive sciences, 17(4), 160-165.

Gandola, M., Invernizzi, P., Sedda, A., Ferrè, E. R., Sterzi, R., Sberna, M., Paulesu, E. \& Bottini, G. (2012). An anatomical account of somatoparaphrenia. Cortex, 48(9), 1165-1178.

Gentile, G., Guterstam, A., Brozzoli, C., \& Ehrsson, H. H. (2013). Disintegration of multisensory signals from the real hand reduces default limb self-attribution: an fMRI study. Journal of Neuroscience, 33(33), 13350-13366.

Gillihan, S. J., \& Farah, M. J. (2005). Is self special? A critical review of evidence from experimental psychology and cognitive neuroscience. Psychological Bulletin, 131(1), 76. 
Haggard, P. (2017). Sense of agency in the human brain. Nature Reviews Neuroscience, 18(4), 196.

Happé, F., Cook, J. L., \& Bird, G. (2017). The structure of social cognition: In (ter) dependence of sociocognitive processes. Annual review of psychology, 68, 243-267.

Hecht, E. E., Patterson, R., \& Barbey, A. K. (2012). What can other animals tell us about human social cognition? An evolutionary perspective on reflexive and reflective processing. Frontiers in Human Neuroscience, 6, 224.

Heyes, C. (2014). Submentalizing: I'm not really reading your mind. Psychological Science. 9, 121-43.

Heyes, C. (2018). Précis of cognitive gadgets: The cultural evolution of thinking. Behavioral and Brain Sciences, 1-57.

Hogeveen, J., Obhi, S. S., Banissy, M. J., Santiesteban, I., Press, C., Catmur, C., \& Bird, G. (2014). Task-dependent and distinct roles of the temporoparietal junction and inferior frontal cortex in the control of imitation. Social Cognitive and Affective Neuroscience, 10(7), 10031009.

Jeannerod, M. (2003). The mechanism of self-recognition in humans. Behavioural brain research, 142(1-2), 1-15.

Just, M. A., Carpenter, P. A., Keller, T. A., Eddy, W. F., \& Thulborn, K. R. (1996). Brain activation modulated by sentence comprehension. Science, 274(5284), 114-116. 
Keenan, J. P., Freund, S., Hamilton, R. H., Ganis, G., \& Pascual-Leone, A. (2000). Hand response differences in a self-face identification task. Neuropsychologia, 38, 1047-1053.

Krall, S. C., Rottschy, C., Oberwelland, E., Bzdok, D., Fox, P. T., Eickhoff, S. B., ... \& Konrad, K. (2015). The role of the right temporoparietal junction in attention and social interaction as revealed by ALE meta-analysis. Brain Structure and Function, 220(2), 587-604.

Kühn, S., Brass, M., \& Haggard, P. (2013). Feeling in control: Neural correlates of experience of agency. Cortex, 49(7), 1935-1942.

Lamm, C., Bukowski, H., \& Silani, G. (2016). From shared to distinct self-other representations in empathy: evidence from neurotypical function and socio-cognitive disorders. Philosophical Transactions of the Royal Society B: Biological Sciences, 371(1686), 20150083.

Lombardo, M. V., Chakrabarti, B., Bullmore, E. T., Wheelwright, S. J., Sadek, S. A., Suckling, J., ... \& Baron-Cohen, S. (2010). Shared neural circuits for mentalizing about the self and others. Journal of cognitive neuroscience, 22(7), 1623-1635.

Mar, R. A. (2011). The neural bases of social cognition and story comprehension. Annual review of psychology, 62, 103-134.

Mars, R. B., Sallet, J., Schüffelgen, U., Jbabdi, S., Toni, I., \& Rushworth, M. F. (2012). Connectivity-based subdivisions of the human right "temporoparietal junction area": evidence for different areas participating in different cortical networks. Cerebral cortex, 22(8), 1894-1903. 
Marsh, L. E., Bird, G., \& Catmur, C. (2016). The imitation game: Effects of social cues on 'imitation'are domain-general in nature. NeuroImage, 139, 368-375.

Mitchell, J. P. (2007). Activity in right temporo-parietal junction is not selective for theoryof-mind. Cerebral cortex, 18(2), 262-271.

Neisser, U. (1995). Criterion for an ecological self. In: Rochat P, editor. The self in infancy: Theory and research. pp. 17-34. Amsterdam: Elsevier

Nieuwenhuys, R., Voogd, J., \& Van Huijzen, C. (2007). The human central nervous system: a synopsis and atlas. Springer Science \& Business Media.

Petkova, V. I., \& Ehrsson, H. H. (2008). If I were you: perceptual illusion of body swapping. PloS one, 3(12), e3832.

Piaget, J., \& Inhelder, B., (1948). The Child's Conception of Space. Routledge and Kegan Paul, London.

Poldrack, R. A. (2006). Can cognitive processes be inferred from neuroimaging data?. Trends in cognitive sciences, 10(2), 59-63.

Poldrack, R. A. (2011). Inferring mental states from neuroimaging data: from reverse inference to large-scale decoding. Neuron, 72(5), 692-697.

Premack, D., \& Woodruff, G. (1978). Does the chimpanzee have a theory of mind? Behavioral and Brain Sciences, 1, 515-526. 
Pulvermüller, F. (2005). Brain mechanisms linking language and action. Nature reviews neuroscience, 6(7), 576.

Quesque, F., Chabanat, E., \& Rossetti, Y. (2018). Taking the point of view of the blind: spontaneous level-2 perspective-taking in irrelevant conditions. Journal of Experimental Social Psychology, 79, 356-364.

Quesque, F. \& Rossetti, Y. (in press). What do theory of mind tasks actually measure? Theory and practice. Perspectives on Psychological Science.

Rogers, C. (1951). Client-centred therapy. Houghton-Mifflin, Boston.

Santiesteban, I., Banissy, M. J., Catmur, C., \& Bird, G. (2015). Functional lateralization of temporoparietal junction-imitation inhibition, visual perspective-taking and theory of mind. European Journal of Neuroscience, 42(8), 2527-2533.

Saxe, R., \& Kanwisher, N. (2003). People thinking about thinking people: the role of the temporo-parietal junction in "theory of mind". Neuroimage, 19(4), 1835-1842.

Saxe R, Wexler A. 2005. Making sense of another mind: the role of the right temporoparietal junction. Neuropsychologia. 43:1391-1399

Schurz, M., Aichhorn, M., Martin, A., \& Perner, J. (2013). Common brain areas engaged in false belief reasoning and visual perspective taking: a meta-analysis of functional brain imaging studies. Frontiers in human neuroscience, $7,712$. 
Schurz, M., Kronbichler, M., Weissengruber, S., Surtees, A., Samson, D. \& Perner, J. (2015). Clarifying the role of theory of mind areas during visual perspective taking: Issues of spontaneity and domain-specificity. NeuroImage, 117, 386-96.

Serences, J. T., Shomstein, S., Leber, A. B., Golay, X., Egeth, H. E., \& Yantis, S. (2005). Coordination of voluntary and stimulus-driven attentional control in human cortex. Psychological science, 16(2), 114-122.

Sierra, M. (2009). Depersonalization: A new look at a neglected syndrome. Cambridge University Press.

Sierra, M., \& Berrios, G. E. (1998). Depersonalization: neurobiological perspectives. Biological psychiatry, 44(9), 898-908.

Silani, G., Lamm, C., Ruff, C. C., \& Singer, T. (2013). Right supramarginal gyrus is crucial to overcome emotional egocentricity bias in social judgments. Journal of neuroscience, 33(39), 15466-15476.

Soutschek, A., Ruff, C. C., Strombach, T., Kalenscher, T., \& Tobler, P. N. (2016). Brain stimulation reveals crucial role of overcoming self-centeredness in self-control. Science advances, 2(10), e1600992.

Sowden, S., \& Catmur, C. (2015). The role of the right temporoparietal junction in the control of imitation. Cerebral Cortex, 25(4), 1107-1113. 
Spengler, S., von Cramon, D. Y., \& Brass, M. (2009). Was it me or was it you? How the sense of agency originates from ideomotor learning revealed by fMRI. Neuroimage, 46(1), 290298.

Sperduti, M., Delaveau, P., Fossati, P., \& Nadel, J. (2011). Different brain structures related to self-and external-agency attribution: a brief review and meta-analysis. Brain Structure and Function, 216(2), 151-157.

Tomova, L., von Dawans, B., Heinrichs, M., Silani, G., \& Lamm, C. (2014). Is stress affecting our ability to tune into others? Evidence for gender differences in the effects of stress on self-other distinction. Psychoneuroendocrinology, 43, 95-104.

Tsakiris, M., \& Haggard, P. (2005). The rubber hand illusion revisited: visuotactile integration and self-attribution. Journal of Experimental Psychology: Human Perception and Performance, 31(1), 80 .

Uddin, L. Q., Molnar-Szakacs, I., Zaidel, E. \& Iacoboni, M. (2006). rTMS to the right inferior parietal lobule disrupts self-other discrimination. Social cognitive and affective neuroscience, $1,65-71$.

Uddin, L. Q. (2011). The self in autism: an emerging view from neuroimaging. Neurocase, 17(3), 201-208. 
Van Ackeren, M. J., Casasanto, D., Bekkering, H., Hagoort, P., \& Rueschemeyer, S. A. (2012). Pragmatics in action: indirect requests engage theory of mind areas and the cortical motor network. Journal of Cognitive Neuroscience, 24(11), 2237-2247.

Van Overwalle, F. (2009). Social cognition and the brain: a meta-analysis. Human brain mapping, 30(3), 829-858.

Vogeley, K., \& Fink, G. R. (2003). Neural correlates of the first-person perspective. Trends in Cognitive Neuroscience, $7,38-42$.

Vogeley, K., Bussfeld, P., Newen, A., Herrmann, S., Happé, F., Falkai, P., ... \& Zilles, K. (2001). Mind reading: neural mechanisms of theory of mind and self-perspective. Neuroimage, 14(1), 170-181.

Wegner, D. M., \& Wheatley, T. (1999). Apparent mental causation: Sources of the experience of will. American psychologist, 54(7), 480.

Wimmer, H., \& Perner, J. (1983). Beliefs about beliefs: Representation and constraining function of wrong beliefs in young children's understanding of deception. Cognition, 13, 103128.

Yarkoni, T., Poldrack, R. A., Nichols, T. E., Van Essen, D. C., \& Wager, T. D. (2011). Large-scale automated synthesis of human functional neuroimaging data. Nature methods, 8(8), 665. 
Young, L., Camprodon, J. A., Hauser, M., Pascual-Leone, A., \& Saxe, R. (2010).

Disruption of the right temporoparietal junction with transcranial magnetic stimulation reduces the role of beliefs in moral judgments. Proceedings of the National Academy of Sciences, 107(15), 6753-6758.

Young, L, Dodell-Feder, D, \& Saxe, R. (2010). What gets the attention of the temporoparietal junction? An fMRI investigation of attention and theory of mind. Neuropsychologia, 48, $2658-2664$.

Zaitchik, D., Walker, C., Miller, S., LaViolette, P., Feczko, E., \& Dickerson, B. C. (2010). Mental state attribution and the temporoparietal junction: an fMRI study comparing belief, emotion, and perception. Neuropsychologia, 48(9), 2528-2536. 


\section{Figure Caption.}

Figure 1. Illustration of the anatomical features of the brain that are encompassed under the label “Temporoparietal Junction" (left hemisphere is here depicted). The TPJ is characterized by the red ellipse. Covered brain regions are represented in orange (supramarginal gyrus), cyan (superior temporal gyrus), green (angular gyrus) and pink (superior temporal sulcus). The Wernicke area is depicted as a blue squared grid.

Figure 2. A. Illustration of the five-dimensional Neisser's (1995) social psychological conception of the self (orange), and the two-dimensional Gillihan and Farah's (2005) neuroscientific conception of the self (green) and their correspondences (double-headed arrow). B. Schematic representation of the different types of self-other distinction mechanisms, grouped according to the aspect of the self that is evaluated.

Figure 3. (Left) Activation in the right TPJ in a classical "sense of agency" task manipulating the congruency between participants' motor performances and displayed visual feedbacks. (Right) Parametric increase of the mean parameter estimates in function of the amount of discrepancy between predicted and actual action-feedbacks over conditions. Inspired from Spengler et al. (2009)

Figure 4. Brain regions selectively activated for the processing of self-other distinction at the mental-state level (from left to right, respectively for "Mental states", "Mentalizing", or "Theory of Mind" processing). The maps have been computed with 'association test' conducted on http://neurosynth.org and modelized on MRIcroGL. The displayed statistical maps, downloaded 
during the spring of 2019, are corrected for multiple comparisons using a false discovery rate criterion of .01. The upper part of the figure displays activations of the left hemisphere while the lower part displays activations of the right hemisphere. 
Figure 1.

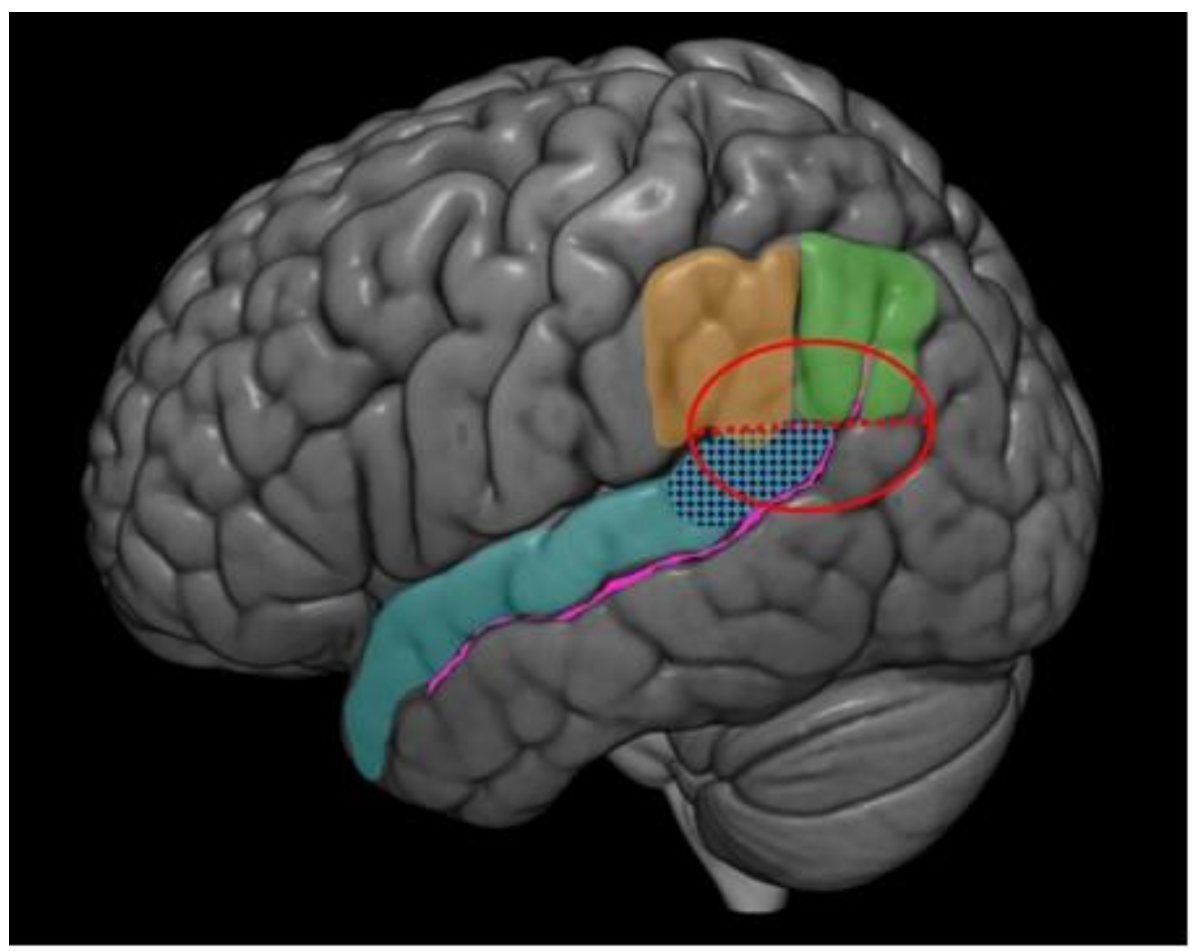


Figure 2.

A

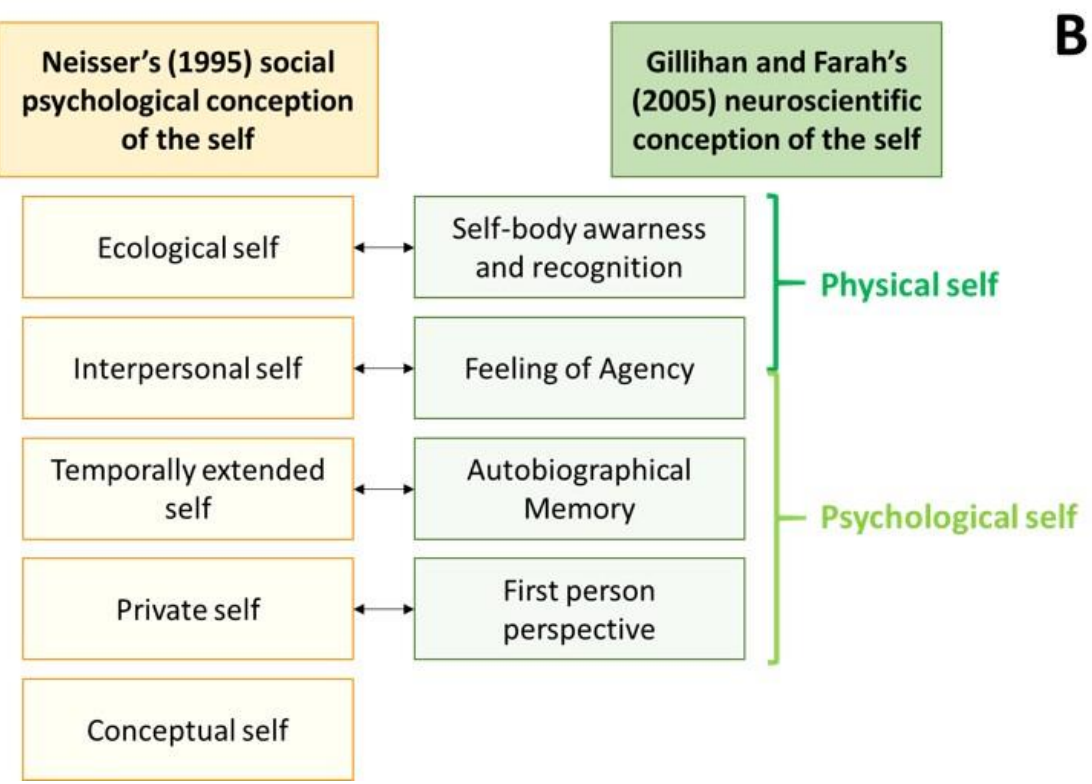

B

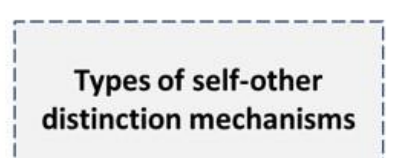

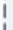

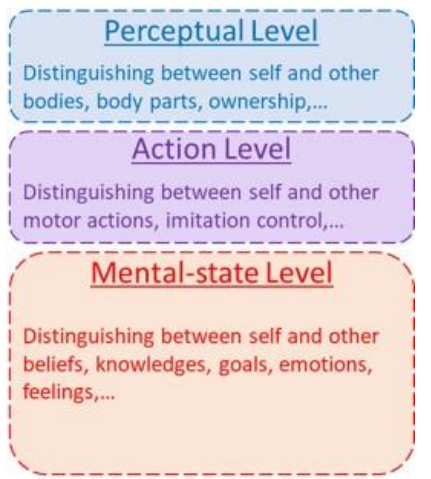


Figure 3.
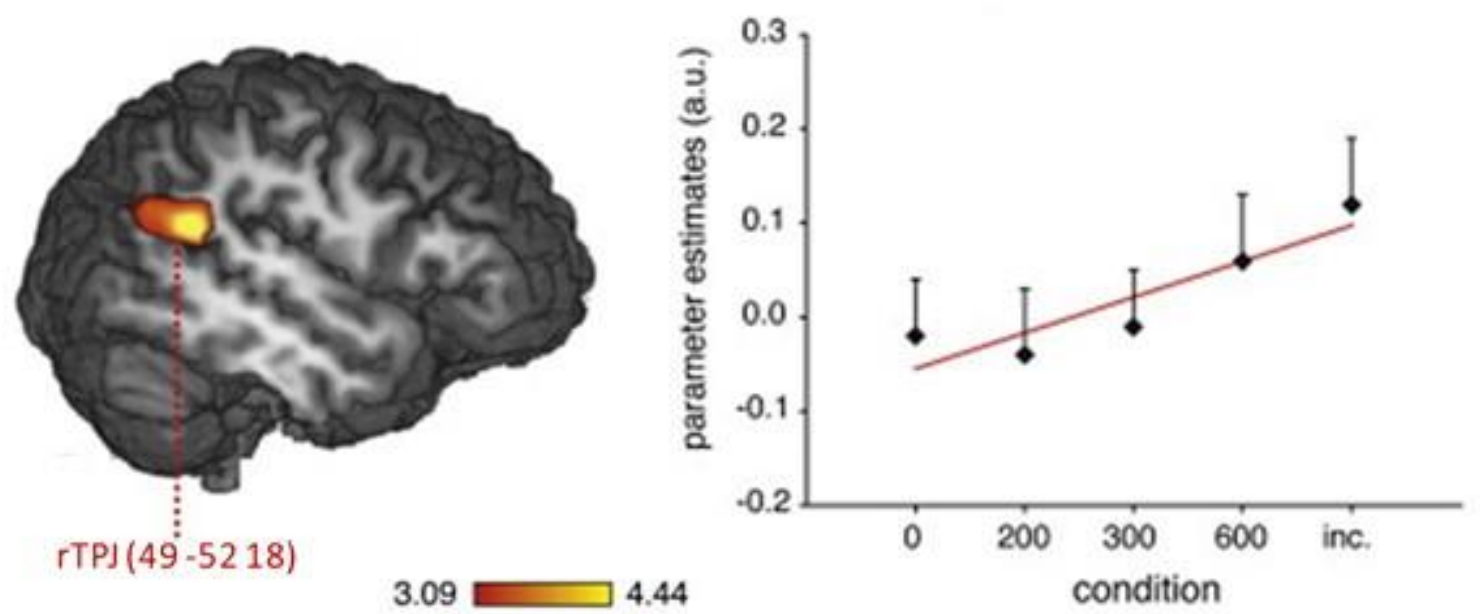
Figure 4.
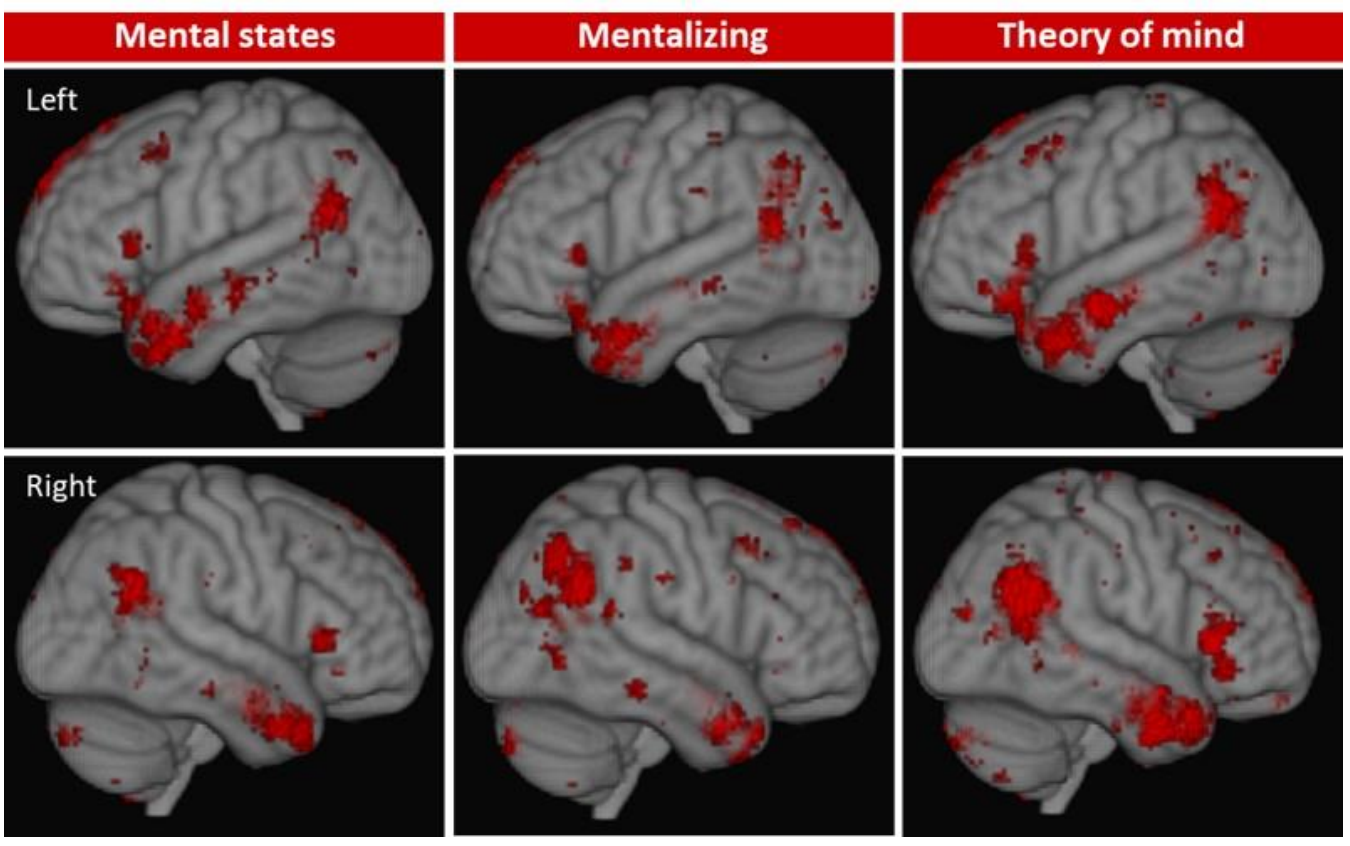